Communications in Physics, Vol. 14, No. 2 (2004), pp. 65-73

\title{
ON THE LOOP EXPANSION OF THE EFFECTIVE ACTION
}

\author{
TRAN HUU PHAT \\ Vietnam Atomic Energy Commission - 59 Ly Thuong Kiet, Hanoi, Vietnam \\ NGUYEN VAN LONG \\ Faculty of Physics, Hanoi University of Education
}

\begin{abstract}
Based on the DeWitt formula the loop expansion of the effective action is easily established. Extending to the system with finite density we develop the in-medium DeWitt formula, which is the starting point for setting up loop expansion of the in-medium effective action.
\end{abstract}

\section{INTRODUCTION}

It is well known that the effective action play a crucial role in quantum field theory, this is because all complete quantum information of a physical system can be extracted from its effective action. Morever, the effective action proves to be very powerful in solving problem relating to the symmetry breaking $[1,2]$ and the symmetry restoration at high temperature. Basically the effective action method is nonperturbative, therefore it is very convenient applying to the phase transition and other nonperturbative phenomena. In this respect, to calculate the effective action of a given quantum system is in the first priority of all studies. There are so far several methods dealing with loop expansion of the effective action [3,4]. However, all of them, perhaps, are cumbersome enough and, therefore, are not very convenient.

In Section 2 of this paper, starting from the old formula of DeWitt [5] we derive the formula for loop expansion of the effective action. Section 3 is devoted to establishing the effective action in medium with non-vanishing density of matter. The conclusion and discussion are given in Section 4.

\section{LOOP EXPANSION FORMULAR}

For the sake of simplicity let us begin with the scalar field described by the Lagrangian:

$$
L=L_{0}[\Phi]+L_{I}[\Phi], \quad \Phi=\left\{\Phi_{1}, \cdots, \Phi_{N}\right\},
$$

in which $\mathrm{L}_{0}$ is the Lagrangian of free fields and $\mathrm{L}_{I}$ corresponds to the interacting fields.

The generating functional for connected Green functions reads

$$
e^{i W[J]}=N \int D \Phi \exp \left\{i \int d x\left[L+J_{k} \Phi_{k}\right]\right\}
$$

where $N$ is the normalisation constant.

The vacuum expectation value of $\Phi_{i}$ in the presence of the external source $\mathrm{J}_{i}(\mathrm{x})$ is given as

$$
\frac{\delta W[J]}{\delta J_{i}(x)}=\left\langle\Phi_{i}(x)\right\rangle_{J}=\varphi_{i}(x)
$$


The effective action $\Gamma[\varphi]$, corresponding to $\varphi(\mathrm{x})$, is defined to be the Legendre transform of $\mathrm{W}[\mathrm{J}]$ :

$$
\Gamma[\phi]=W[J]-\int d x J_{i}(x) \varphi_{i}(x)
$$

In favour of (2) it is clear that

$$
\frac{\delta \Gamma[\phi]}{\delta \varphi_{i}(x)}=-J_{i}(x)
$$

This is the equation of motion for $\varphi(x)$, containing only classical quantities (c-number).

Next, let us introduce formally the classical action $S_{J}[\Phi]$ in the presence of external sourse $J(x)$ :

$$
\begin{gathered}
S_{J}[\Phi]=\int d x\left[L[\Phi]+J_{i}(x) \Phi_{i}(x)\right] \\
=S[\Phi]+\int d x J_{i}(x) \Phi_{i}(x)
\end{gathered}
$$

where $\mathrm{S}[\Phi]$ is the conventional classical action,

$$
S[\Phi]=\int d x L[\Phi]
$$

From the equation

$$
\frac{\delta S_{J}[\Phi]}{\delta \Phi_{i}(x)}=0
$$

it follows that

$$
\frac{\delta S[\Phi]}{\delta \Phi_{i}(x)}=-J_{i}(x)
$$

which is the equations of operators (q-number).

Combining (4) and (5) we arrive at

$$
\frac{\delta \Gamma[\phi]}{\delta \varphi_{i}(x)}=\left\langle\frac{\delta S[\Phi]}{\delta \Phi_{i}(x)}\right\rangle
$$

which is the DeWitt formula, connecting the quantum action with the classical one.

Making use of the formula for vacuum expectation value of an arbitrary operator [6] we can rewrite (6) as

$$
\frac{\delta \Gamma[\phi]}{\delta \varphi_{i}(x)}=: \exp \left\{\frac{i}{\hbar} \sum_{n=2}^{\infty} \frac{(-i \hbar)^{n}}{n !} \operatorname{Tr} G_{\left(x_{1} \cdots x_{n}\right)}^{i_{1} \cdots i_{n}} \frac{\delta^{n}}{\delta \varphi_{i_{1}}\left(x_{1}\right) \cdots \delta \varphi_{i_{n}}\left(x_{n}\right)}\right\}: \frac{\delta S[\phi]}{\delta \varphi_{i}(x)}
$$

where $\operatorname{Tr}$ denotes the summation over repeated discrete indices and/or the integration over repeated continuous arguments.

Equation (7) contains two unknown quantities: effective action $\Gamma$ and Green functions G. Therefore, we need another equation connecting them. For this end, let us take derivative of (2) and (4) with respect to $\mathrm{J}_{k}$ and $\varphi_{j}$, respectively. We get

$$
\operatorname{Tr} \frac{\delta^{2} \Gamma[\phi]}{\delta \varphi_{i}(x) \delta \varphi_{j}(z)} \frac{\delta^{2} W[J]}{\delta J_{j}(z) \delta J_{k}(y)}=-\delta_{i k} \delta(x-y)
$$


or

$$
\operatorname{Tr} G_{i j}(x, z) \frac{\delta^{2} \Gamma[\varphi]}{\delta \varphi_{j}(z) \delta \varphi_{k}(y)}=-\delta_{i k} \delta(x-y)
$$

Now let us prove the formula for loop expansion of the effective action

$$
\Gamma[\varphi]=S[\varphi]+\frac{i}{2} \operatorname{Tr} \ln D_{i k}+\Gamma^{(2)},
$$

in which $D_{i k}^{-1}=\left.\frac{\delta^{2} S[\Phi]}{\delta \Phi_{i} \delta \Phi_{k}}\right|_{\Phi=\varphi}$ and $\Gamma^{(2)}$ is the sum of all one-particle-irreducible (1PI) vacuum graphs, in which internal lines represent the propagator $\mathrm{D}_{i k}$ and vertices are determined by the shifting Lagrangian $L[\Phi+\varphi]$.

The proof is carried out as follows. Assume the loop expansions for $\Gamma$ and $G$ have the general form

$$
\begin{aligned}
\Gamma & =\Gamma_{0}+\hbar \Gamma_{1}+\hbar^{2} \Gamma_{2}+\cdots \\
G & =G_{0}+\hbar G_{1}+\hbar^{2} G_{2}+\cdots
\end{aligned}
$$

Substituting (10) and (11) into (7) and (8), respectively, we receive successively

$$
\begin{gathered}
\frac{\delta \Gamma_{0}}{\delta \varphi_{i}}=\frac{\delta S}{\delta \varphi_{i}} \\
\frac{\delta \Gamma_{1}}{\delta \varphi_{i}(x)}=-\frac{i}{2} \operatorname{Tr} G_{0}^{i_{1} i_{2}}\left(x_{1}, x_{2}\right) \frac{\delta}{\delta \varphi_{i}(x)} \frac{\delta^{2} S}{\delta \varphi_{i_{1}}\left(x_{1}\right) \delta \varphi_{i_{2}}\left(x_{2}\right)} \\
\frac{\delta \Gamma_{2}}{\delta \varphi_{i}(x)}=: \\
\left\{-\frac{i}{2} \operatorname{Tr} G_{1}^{i_{1} i_{2}}\left(x_{1}, x_{2}\right) \frac{\delta^{2}}{\delta \varphi_{i_{1}}\left(x_{1}\right) \delta \varphi_{i_{2}}\left(x_{2}\right)}-\right. \\
\left.\frac{1}{3 !} \operatorname{Tr} G_{0}^{i_{1} i_{2} i_{3}}\left(x_{1}, x_{2}, x_{3}\right) \frac{\delta^{3}}{\delta \varphi_{i_{1}}\left(x_{1}\right) \delta \varphi_{i_{2}}\left(x_{2}\right) \delta \varphi_{i_{3}}\left(x_{3}\right)}\right\}: \frac{\delta S}{\delta \varphi_{i}(x)}
\end{gathered}
$$

and

$$
\begin{gathered}
\operatorname{Tr} G_{0}^{i j}\left(x_{i}, x_{j}\right) \frac{\delta^{2} \Gamma_{0}}{\delta \varphi_{j}\left(x_{j}\right) \delta \varphi_{k}\left(x_{k}\right)}=-\delta_{i k} \delta\left(x_{i}-x_{k}\right) \\
\operatorname{Tr}\left\{G_{1}^{i j}\left(x_{i}, x_{j}\right) \frac{\delta^{2} \Gamma_{0}}{\delta \varphi_{j}\left(x_{j}\right) \delta \varphi_{k}\left(x_{k}\right)}+G_{0}^{i j}\left(x_{i}, x_{j}\right) \frac{\delta^{2} \Gamma_{1}}{\delta \varphi_{j}\left(x_{j}\right) \delta \varphi_{k}\left(x_{k}\right)}\right\}=0
\end{gathered}
$$

Integrating (12) over $\varphi_{i}$ gives immediately

$$
\Gamma_{0}[\varphi]=S[\varphi]
$$

which together with (15) provides

$$
G_{0, i k}=D_{i k}
$$

Inserting (18) into (13), we obtain

$$
\frac{\delta \Gamma_{1}}{\delta \varphi_{i}}=\frac{i}{2} \operatorname{Tr} D_{j k}^{-1} \frac{\delta D_{j k}}{\delta \varphi_{i}}
$$


which leads to

$$
\Gamma_{1}[\varphi]=\frac{i}{2} \operatorname{Tr} \ln D_{j k}
$$

Next let us prove that $\Gamma_{2}$ determined by (14) is the sum of two-loop vacuum graphs with internal lines defined by $\mathrm{D}_{i k}$ and vertices defined by $\mathrm{S}[\Phi+\varphi]$.

Indeed, it is easily seen that $G_{1}^{i k}$ and $G_{0}^{i j k}$ can be expressed in terms of $\mathrm{G}_{0, i k}\left(=\mathrm{D}_{i k}\right)$. From (16) it follows that

$$
G_{1}^{i k}=-G_{0}^{i j} \frac{\delta^{2} \Gamma_{1}}{\delta \varphi_{j} \delta \varphi_{m}} G_{0}^{m k}
$$

On the other hand,

$$
G^{i j k}=\left.\frac{\delta}{\delta J_{i}} \frac{\delta^{2} W}{\delta J_{j} \delta J_{k}}\right|_{J=0}=\left.\frac{\delta}{\delta J_{i}} G^{j k}\right|_{J=0}=G^{i l} G^{j m} G^{k n} \frac{\delta^{3} \Gamma}{\delta \varphi_{l} \delta \varphi_{m} \delta \varphi_{n}}
$$

Inserting (10) and (11) into (21) we get

$$
G_{0}^{i j k}=G_{0}^{i l} G_{0}^{j m} G_{0}^{k n} \frac{\delta^{3} \Gamma_{0}}{\delta \varphi_{l} \delta \varphi_{m} \delta \varphi_{n}}
$$

Finally, substituting (20) and (22) into (14) we arrive at an expression containing only $D_{i k}$, which represents internal lines, and variational differentials of $\mathrm{S}[\varphi]$, which corresponds to vertices.

The similiar discussion can be further applied, respectively, to $\Gamma_{3}, \Gamma_{4}$ and so on. We completed the proof of (9).

As an illustration, let us apply the formula (9) to calculation of the effective action for the linear sigma model in two-loop approximation.

The Lagrangian of this model reads

$$
L=\frac{1}{2}\left[\left(\partial_{\mu} \sigma\right)^{2}+\left(\partial_{\mu} \vec{\pi}\right)^{2}\right]-\frac{\mu^{2}}{2}\left[\sigma^{2}+\vec{\pi}^{2}\right]-\frac{\lambda^{2}}{4}\left[\sigma^{2}+\vec{\pi}^{2}\right]^{2}
$$

The positivity of the Hamiltonian corresponding to (23) demands that $\lambda^{2}>0$. We shall choose $\lambda>0$.

Let $v$ be the vacuum expectation value of $\sigma:\langle\sigma\rangle=v \neq 0$ and $\langle\vec{\pi}\rangle=0$. The field $\sigma$ is shifted $\sigma=s+v$ so that $\langle s\rangle=0$.

We shall now rewrite (23) in terms of $\mathrm{s}$

$$
\begin{aligned}
& L=L_{a}+L_{b} \\
& L_{a}=\frac{1}{2}\left[\left(\partial_{\mu} \vec{\pi}\right)^{2}-\mu_{\pi}^{2} \vec{\pi}^{2}\right]+\frac{1}{2}\left[\left(\partial_{\mu} s\right)^{2}-\mu_{\sigma}^{2} s^{2}\right]-\lambda^{2} v s\left(s^{2}+\vec{\pi}^{2}\right)-\frac{\lambda^{2}}{4}\left(s^{2}+\vec{\pi}^{2}\right)^{2}, \\
& L_{b}=-v \mu_{\pi}^{2} s
\end{aligned}
$$

We have dropped an inessential c-number constant in the expression given above. We have also used the abbreviations

$$
\mu_{\pi}^{2}=\mu^{2}+\lambda^{2} v^{2}
$$




$$
\mu_{\sigma}^{2}=\mu^{2}+3 \lambda^{2} v^{2}
$$
by

The propagators of free $\sigma$ and $\pi$ fields, corresponding to (24), are given respectively

$$
D_{i k}(p)=\frac{1}{p^{2}-\mu_{\pi}^{2}} \delta_{i k} \quad \Delta(p)=\frac{1}{p^{2}-\mu_{\sigma}^{2}}
$$

in momentum space.

It is known that for $\mathrm{v}=$ const, the effective action is reduced to the effective potential:

$$
\Gamma[v]=-V[v] \int d x
$$

Then, taking into account (25), it follows from (9) that

$$
V[v]=\frac{\mu^{2}}{2} v^{2}+\frac{\lambda^{2}}{4} v^{4}+\frac{i}{2} \int \frac{d^{4} k}{(2 \pi)^{4}} \ln \left(k^{2}-\mu_{\pi}^{2}\right)+\frac{i}{2} \int \frac{d^{4} k}{(2 \pi)^{4}} \ln \left(k^{2}-\mu_{\sigma}^{2}\right)+V_{2},
$$

where $V_{2}$ is the sum of all 1PI vacuum graphs, which are depicted in Fig. 1. in the two-loop approximation,

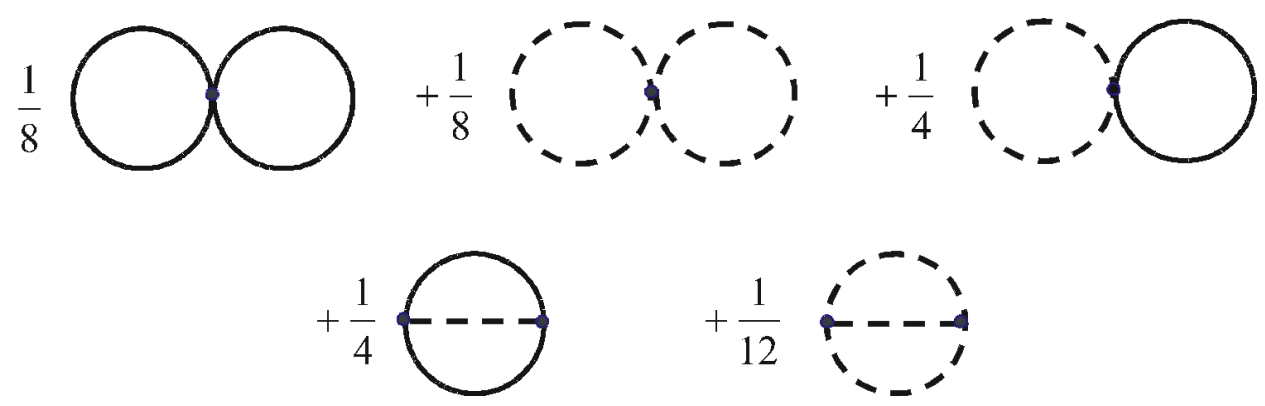

Fig. 1. Two-loop vacuum graphs contribute to $\mathrm{V}_{2}$. The solid line corresponds to $D_{i k}$, the dotted line $-\Delta$. The $\pi^{4}$-vertex corresponds to $-2 i \lambda^{2}\left(\delta_{i k} \delta_{l m}+\delta_{i l} \delta_{k m}+\delta_{i m} \delta_{k l}\right)$, the $\sigma^{4}$-vertex $-6 i \lambda^{2}$, the $\sigma^{2} \pi^{2}$-vertex $-2 i \lambda^{2} \delta_{i k}$, the $\sigma \pi^{2}$-vertex $-2 i \lambda^{2} v \delta_{i k}$ and the $\sigma^{3}$-vertex $-6 i \lambda^{2} v$.

$$
\begin{aligned}
V_{2}=\frac{3}{4} i \lambda^{2} & {\left[\int \frac{d^{4} p}{p^{2}-\mu_{\sigma}^{2}}\right]^{2}+\frac{27}{4} i \lambda^{2}\left[\int \frac{d^{4} p}{p^{2}-\mu_{\pi}^{2}}\right]^{2}+\frac{9}{2} i \lambda^{2} \int \frac{d^{4} p}{p^{2}-\mu_{\pi}^{2}} \int \frac{d^{4} k}{k^{2}-\mu_{\sigma}^{2}} } \\
& +3 \lambda^{4} v^{2} \int \frac{d^{4} p}{(2 \pi)^{4}} \int \frac{d^{4} q}{(2 \pi)^{4}} \frac{1}{p^{2}-\mu_{\pi}^{2}} \cdot \frac{1}{q^{2}-\mu_{\pi}^{2}} \cdot \frac{1}{(p+q)^{2}-\mu_{\sigma}^{2}} \\
& +3 \lambda^{4} v^{2} \int \frac{d^{4} p}{(2 \pi)^{4}} \int \frac{d^{4} q}{(2 \pi)^{4}} \frac{1}{p^{2}-\mu_{\sigma}^{2}} \cdot \frac{1}{q^{2}-\mu_{\sigma}^{2}} \cdot \frac{1}{(p+q)^{2}-\mu_{\sigma}^{2}} .
\end{aligned}
$$




\section{IN-MEDIUM EFFECTIVE ACTION}

Now let us generalize the result obtained above to the system with non-vanishing density of matter. For convenience, let us start from the nuclear matter, described by the Walecka model, whose Lagrangian reads

$$
\begin{aligned}
& L=-\bar{\Psi}\left[\gamma_{\mu} \partial_{\mu}+M\right] \Psi-\frac{1}{2}\left[\partial_{\mu} \sigma \partial_{\mu} \sigma+m_{s}^{2} \sigma^{2}\right]-\frac{1}{4} F_{\mu \nu} F_{\mu \nu} \\
& \quad-\frac{1}{2} m_{v}^{2} A_{\mu}^{2}+g_{s} \bar{\Psi} \sigma \Psi+i g_{v} \bar{\Psi} \hat{A} \Psi
\end{aligned}
$$

where $\mathrm{F}_{\mu \nu}=\partial_{\mu} \mathrm{A}_{\nu}-\partial_{\nu} \mathrm{A}_{\mu} ; \Psi, \sigma$ and $\mathrm{A}_{\mu}$ are the fields operators of nucleon, sigma and vector mesons, respectively; $\mathrm{g}_{s}$ and $\mathrm{g}_{v}$ are coupling constants; $\mathrm{M}, \mathrm{m}_{s}$ and $\mathrm{m}_{v}$ are physical masses of nucleon and mesons, respectively; $\gamma_{\mu}$ satisfy

$$
\gamma_{\mu} \gamma_{\nu}+\gamma_{\nu} \gamma_{\mu}=\delta_{\mu \nu}
$$

A 4-vector is defined as $\mathrm{A}_{\mu}=\left(\mathrm{A}_{k}, \mathrm{~A}_{4}\right)=\left(\mathrm{A}_{k}, \mathrm{iA}_{0}\right), \mathrm{k}=1,2,3$; a scalar product of 4-vectors is $\mathrm{A}_{\mu} \mathrm{B}_{\mu}=\mathrm{A}_{i} \mathrm{~B}_{i}+\mathrm{A}_{4} \mathrm{~B}_{4}=\mathrm{A}_{i} \mathrm{~B}_{i}-\mathrm{A}_{0} \mathrm{~B}_{0}$. Let $\rho$ be the nuclear density, the in-medium propagator of non-interacting nucleon is

$$
S_{0}(p)=(\hat{p}-M)^{-1}+2 i \pi \delta\left(p^{0}-E_{k}\right) \theta\left(p_{F}-|\vec{p}|\right)(\hat{p}+M) / 2 E(p)
$$

in which

$$
\begin{aligned}
& p_{F}^{3}=N_{c} N_{f} 4 \rho /(3 \pi)^{2} \\
& E(p)=\left(\vec{p}^{2}+M^{2}\right)^{1 / 2}
\end{aligned}
$$

Then the generating functional for in-medium connecting Green functions is defined as

$$
\begin{aligned}
e^{i W_{\rho}\left[\bar{\eta}, \eta, J, J_{\mu}\right]}= & N \int D \bar{\Psi} D \Psi D \sigma D A_{\mu} \times \\
& \exp i\left[\int d x\left[L(\rho)+\bar{\eta} \Psi+\bar{\Psi} \eta+J \sigma+J_{\mu} A_{\mu}\right]\right]
\end{aligned}
$$

where $\mathrm{L}(\rho)$ is the Lagrangian of system at density $\rho$, obtained by substituting (27) into $(26)$, i.e.

$$
L(\rho)=\bar{\Psi}\left[S_{0}^{-1}(x)+g_{s} \sigma(x)+i g_{v} \hat{A}\right] \Psi+\text { meson part }
$$

$S_{0}(x)$ is the Fourier transform of $S_{0}(p)$. (28) gives immediately the expectation values of field operators in presence of external sources and at finite density,

$$
\begin{gathered}
\frac{\delta W_{\rho}}{\delta \bar{\eta}(x)}=\langle\Psi(x)\rangle \quad ; \quad \frac{\delta W_{\rho}}{\delta \eta(x)}=\langle\bar{\Psi}(x)\rangle \\
\frac{\delta W_{\rho}}{\delta J(x)}=\langle\sigma(x)\rangle=v ; \quad \frac{\delta W_{\rho}}{\delta J_{\mu}(x)}=\left\langle A_{\mu}(x)\right\rangle=\omega_{\mu}
\end{gathered}
$$

The effective action at density $\rho$ is defined to be the Legendre transform of $W$ :

$$
\Gamma_{\rho}\left[\langle\bar{\Psi}\rangle,\langle\Psi\rangle, v, \omega_{\mu}\right]=W_{\rho}\left[\bar{\eta}, \eta, J, J_{\mu}\right]-\int d x\left[\bar{\eta}\langle\Psi\rangle+\langle\bar{\Psi}\rangle \eta+J\langle\sigma\rangle+J_{\mu}\left\langle A_{\mu}\right\rangle\right]
$$


From (29) it follows that

$$
\begin{gathered}
\frac{\delta \Gamma_{\rho}}{\delta\langle\bar{\Psi}(x)\rangle}=-\eta(x) ; \quad \frac{\delta \Gamma_{\rho}}{\delta\langle\Psi(x)\rangle}=-\bar{\eta}(x) \\
\frac{\delta \Gamma_{\rho}}{\delta v(x)}=-J(x) ; \quad \frac{\delta \Gamma_{\rho}}{\delta \omega_{\mu}(x)}=-J_{\mu}(x)
\end{gathered}
$$

The ground state is corresponding to (30) with vanishing external sources $\bar{\eta}=\eta=$ $J=J_{\mu}=0$. In this case it is clear that $\langle\Psi\rangle=\langle\bar{\Psi}\rangle=0, \mathrm{v} \neq 0$ and $\omega_{\mu}=\delta_{0 \mu} \omega, \omega \neq 0$.

Next let us establish formally the classical action at finite density in the presence of external sources

$$
\begin{aligned}
S_{J}\left[\rho ; \bar{\Psi}, \Psi, \sigma, A_{\mu}\right] & =S\left[\rho ; \bar{\Psi}, \Psi, \sigma, A_{\mu}\right]+\int d x\left[\bar{\eta} \Psi+\bar{\Psi} \eta+J \sigma+J_{\mu} A_{\mu}\right], \\
S\left[\rho ; \bar{\Psi}, \Psi, \sigma, A_{\mu}\right] & =\int d x L(\rho)
\end{aligned}
$$

It is easily seen that from $\delta S_{J}=0$ we get

$$
\begin{gathered}
\frac{\delta S\left[\rho ; \bar{\Psi}, \Psi, \sigma, A_{\mu}\right]}{\delta F(x)}=-E(x), \\
F=\left\{\bar{\Psi}, \Psi, \sigma, A_{\mu}\right\}, \quad E=\left\{\bar{\eta}, \eta, J, J_{\mu}\right\} .
\end{gathered}
$$

Confronting (32) with (30) we arrive at the DeWitt formula at density $\rho$ :

$$
\begin{gathered}
\frac{\delta \Gamma_{\rho}}{\delta \bar{F}(x)}=\left\langle\frac{\delta S\left[\rho ; \bar{\Psi}, \Psi, \sigma, A_{\mu}\right]}{\delta F(x)}\right\rangle, \\
F=\left\{\bar{\Psi}, \Psi, \sigma, A_{\mu}\right\}, \quad \bar{F}=\left\{\langle\bar{\Psi}\rangle,\langle\Psi\rangle,\langle\sigma\rangle,\left\langle A_{\mu}\right\rangle\right\} .
\end{gathered}
$$

Basing on (33) we derive the loop expansion for in-medium effective action $\Gamma_{\rho}$ :

$$
\begin{aligned}
\Gamma_{\rho}=S[ & \left.\rho ;\langle\bar{\Psi}\rangle,\langle\Psi\rangle,\langle\sigma\rangle,\left\langle A_{\mu}\right\rangle\right]+\frac{1}{2} \operatorname{Tr} \ln C^{-1}(x)+\frac{1}{2} \operatorname{Tr} \ln D_{\mu \nu}^{-1}(x) \\
& -\operatorname{Tr} \ln G^{-1}\left(x ; v, \omega_{\mu}\right)+\Gamma_{2}
\end{aligned}
$$

where

$$
\begin{gathered}
C_{0}^{-1}(x)=\frac{\delta^{2} S\left[\rho ; \bar{\Psi}, \Psi, \sigma, A_{\mu}\right]}{\delta \sigma(x) \delta \sigma(0)}, \quad D_{0 \mu \eta}^{-1}(x)=\frac{\delta^{2} S\left[\rho ; \bar{\Psi}, \Psi, \sigma, A_{\mu}\right]}{\delta A_{\mu}(x) \delta A_{\nu}(0)}, \\
G_{0}^{-1}\left(x ; v, \omega_{\mu}\right)=\frac{\delta^{2} S\left[\rho ; \bar{\Psi}, \Psi, \sigma, A_{\mu}\right]}{\delta \bar{\Psi}(x) \delta \Psi(0)} \mid \begin{array}{l}
\sigma=v, \quad, \\
A_{\mu}=\delta_{\mu \eta} \omega
\end{array}
\end{gathered}
$$

$\Gamma_{2}$ is the sum of all 1PI vacuum graphs which, in two-loop approximation, are given in Fig. 2. 

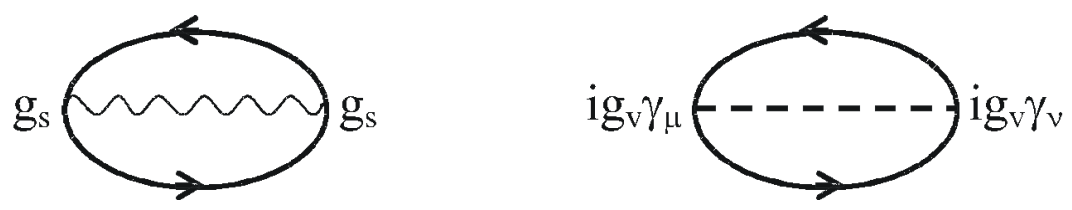

Fig. 2. Solid line represents the nucleon propagator $G$, wavy line-propagator of sigma meson $C$ and dotted line-propagator of vector meson $D_{\mu \nu}$.

Because $v$ and $\omega$ are not dependent on space-time coordinates, the theory is invariant with respect to translations. So, instead of (3.9) we can write the effective potential $V_{\rho}$,

$$
\Gamma_{\rho}=-V_{\rho} \int d x
$$

in momentum space,

$$
\begin{gathered}
V_{\rho}[v, \omega]=-\frac{m_{s}^{2}}{2} v^{2}-\frac{m_{v}^{2}}{2} \omega^{2}+\frac{1}{2} \int \frac{d^{4} k}{(2 \pi)^{4}} \ln \left(k^{2}+m_{s}^{2}\right) \\
+\frac{1}{2} \int \frac{d^{4} k}{(2 \pi)^{4}} \ln \left(k^{2}+m_{v}^{2}\right)+\frac{1}{2} \int \frac{d^{4} k}{(2 \pi)^{4}} G(v) \\
+\frac{i}{2} g_{s}^{2} \int \frac{d^{4} p}{(2 \pi)^{4}} \int \frac{d^{4} k}{(2 \pi)^{4}} \operatorname{tr} G(p) G(k) C(p-k) \\
-\frac{i}{2} g_{v}^{2} \int \frac{d^{4} p}{(2 \pi)^{4}} \int \frac{d^{4} k}{(2 \pi)^{4}} \operatorname{tr} \gamma_{\mu} G(p) \gamma_{\nu} G(k) D_{\mu \eta}(p-k) .
\end{gathered}
$$

where

$$
\begin{gathered}
C_{0}(k)=-\left(k^{2}+m_{s}^{2}\right)^{-1}, \\
D_{0, \mu \eta}(k)=-\left(k^{2}+m_{v}^{2}\right)^{-1}\left(\delta_{\mu \eta}+\frac{k_{\mu} k_{\nu}}{m_{v}^{2}}\right), \\
G_{0}(k)=G_{F}^{0}(k)+G_{D}^{0}(k), \\
G_{F}^{0}(k)=\left[-i \gamma_{\mu} k_{\mu}^{*}+M^{*}\right] \frac{-1}{k^{*^{2}}+M^{*^{2}}} \\
G_{D}^{0}(k)=\left[-\gamma_{\mu} k_{\mu}^{*}+M^{*}\right] \frac{\pi_{i}}{E^{*}(k)} \delta\left(k_{0}^{*}-E^{*}(k)\right) \theta\left(k_{0}\right) \theta\left(k_{F}-|\vec{k}|\right),
\end{gathered}
$$

and $k_{i}^{*}=k_{i}, k_{0}^{*}=k_{0}+g_{v} \omega, \quad M^{*}=M-g_{0} \sigma, E^{*}(k)=\left(\vec{k}^{*^{2}}+M^{*^{2}}\right)^{1 / 2}$.

The expression (35) for in-medium effective potential $V_{\rho}$ is useful for study of nuclear matter.

\section{CONCLUSION AND DISCUSSION}

In the preceeding sections we developed a new approach for loop expansion of the effective action in vacuum and in medium. It would be noticed that this is Jackiw [3], 
who is the first to establish the formula (9) long ago, but his proof is too lengthy and cumbersome. Basing on the well known formula of DeWitt we obtain (9) rather simply. Generalizing to the in-medium case we get the in-medium DeWitt formula (33), which is the starting point for receiving the loop expansion of the in-medium effective action (35). These are our major results, which are very useful for the study of many non-perturbative phenomena occurred in vacuum as well as in medium such as the spontaneuos breakdown of symmetry in vacuum and in nuclear matter.

\section{ACKNOWLEDGEMENT}

This paper is financially supported by the National Program for Fundamental Reseach of Vietnam and the Vietnam Atomic Energy Commission.

\section{REFERENCES}

1. R. Fukuda, M. Komachiya, S. Yokojima, Y. suzuki, K. Okumura and T. Inagaki, Prog. Theoretical Physics, Supplement, 121(1995) 1-427.

2. L. Dolan and R. Jackiw, Phys.Rev., D9 (1974) 3320.

3. R. Jackiw, Phys.Rev., D9 (1974) 1686.

4. B.A. Faizullaev and M.M Musakhanov, Annals of Physics, 241(1995) 394.

5. B.S. DeWitt, Dynamical Theory of Groups and Fields, Gordon and Breach Publi-sher, New York 1965.

6. A.N. Vassiliev, Functional Methods in Quantum Theory of Field and Statistics, Leningrad University Publisher, 1976 (in Russian). 